\title{
A COUNTEREXAMPLE IN A UNIQUE CONTINUATION PROBLEM
}

\author{
THOMAS H. WOLFF
}

The purpose of this paper is to prove the following result.

Theorem 1. (a) If $d \geq 4$ there is a smooth function $u: \mathbb{R}^{d} \rightarrow \mathbb{R}$, not identically zero, which vanishes to infinite order at the origin and satisfies $|\triangle u(x)| \leq C|x|^{-1}|\nabla u(x)|$ for a certain constant $C$.

(b) If $d \geq 5$ then the function $u$ in (a) may be taken so that in addition $|\triangle u| \leq V|\nabla u|$ with $V \in L^{d}$.

Part (b) complements work of Jerison-Kenig [3] and the author [6, 8]. Namely, the analogous question for Schrodinger type inequalities is whether there can be a function satisfying $|\triangle u| \leq V|u|$ with $V \in L^{d / 2}$ and vanishing to infinite order at the origin, and in [3] this is shown to be impossible in all dimensions $d \geq 3$. In [8] it is shown that there is no function satisfying the inequality of (b) and vanishing on an open set, and in [6] it is shown that if $d \leq 4$ there is no function satisfying this inequality and vanishing to infinite order at a point. Analogous positive results are also known for the inequality in (a): see for example Pan [4] where it is shown that there is no function vanishing to infinite order at the origin and satisfying $|\triangle u| \leq C|x|^{-2}|u|$, and also that examples as in (a) are impossible when $d=2$. Note that we leave it open whether such examples exist when $d=3$ and more significantly do not answer the question of what is the best $L^{p}$ exponent to replace $\mathrm{d}$ in (b). It's not clear to us whether $d+\epsilon$ should work or whether possibly the exponent $\frac{3 d-4}{2}$ obtained in [6] is optimal. There is a procedure going back to Plis (e.g. [5]) and P. Cohen for constructing such counterexamples and we will follow this procedure here, at least in principle. It can be thought of as taking place 
in two stages: a finite construction followed by an iteration. Section 1 of this paper contains the finite construction and Section 2 contains the iteration. We will use the notation $x \lesssim y$ to mean that $x \leq C y$ where $\mathrm{C}$ is a constant depending only on the dimension or other clearly specified quantities, and $x \approx y$ for " $x \lesssim y$ and $y \lesssim x "$.

We are expending quite a lot of effort to gain comparatively little, since it is easy (in any $\mathbb{R}^{d}$ ) to find functions $u: \mathbb{R}^{d} \rightarrow \mathbb{R}$ vanishing to infinite order at the origin and such that $\frac{\Delta u}{|\nabla u|} \in L^{p}$ for all $p<d$. On the other hand, in order to prove Theorem 1 it is necessary to work with highly oscillatory functions, for reasons which are discussed at the end of [7], and it seems unlikely (to the author at least) that there is a way of doing this which does not involve a fair amount of calculation.

Acknowledgement. I thank Wensheng Wang for comments on a preliminary version of this paper.

\section{Proof of Theorem 1, Part 1.}

In this section we prove Lemma 1.1 stated below.

We always assume $d \geq 3$, and denote variables in $\mathbb{R}^{d}$ by $x=(\bar{x}, \overline{\bar{x}})$ with $\bar{x} \in \mathbb{R}^{2}$ and $\overline{\bar{x}} \in \mathbb{R}^{d-2}$. We identify $\bar{x}$ with the complex number $x_{1}+i x_{2}$, and define $r, \theta$ and $\rho$ by $\bar{x}=(r \cos \theta, r \sin \theta)$ and $|\overline{\bar{x}}|=\rho$. We let $\alpha$ and $\beta$ be two small positive constants to be specified later and define $A_{n}=\left\{x \in \mathbb{R}^{d}\right.$ : $\left.1-\alpha \leq r \leq 1+\alpha, \rho \leq \beta n^{-1 / 2}\right\}$.

Lemma 1.1. Assume $d \geq 4$. If $n$ is sufficiently large then there is a smooth function $u_{n}: \mathbb{R}^{d} \rightarrow \mathbb{R}$ which is even if $n$ is even and odd if $n$ is odd, and such that

(i) $u_{n}(x)=\mathcal{O}\left(|x|^{n}\right)$ at $\infty, u_{n}(x)=\mathcal{O}\left(|x|^{n+2}\right)$ at 0 .

(ii) supp $\triangle u_{n} \subset A_{n}$.

(iii) $\left|\triangle u_{n}\right| \lesssim r^{n}$ on $A_{n}$.

(iv) $\left|\nabla u_{n}\right| \gtrsim r^{n}$ on $A_{n}$.

Remark. 1) The constants in (iii) and (iv) are of course independent of $n$. 
2) The "shape" of the $A_{n}$ (two long sides, $d-2$ short sides) should be compared to known counterexamples involving Carleman inequalities and related oscillatory integrals, e.g. [1, 2].

3) An immediate consequence of (ii), (iii), (iv) is that $\left\|\frac{\Delta u_{n}}{\left|\nabla u_{n}\right|}\right\|_{d} \lesssim n^{-\frac{1}{2}(d-2)}$. This is best possible, in the sense that there is a constant $\mathrm{C}$ such that any function $u_{n}$ with $\Delta u_{n} \in C_{0}^{\infty}\left(\mathbb{R}^{d} \backslash\{0\}\right)$ and satisfying (i) must also satisfy $\left\|\frac{\Delta u_{n}}{|\nabla u n|}\right\|_{d} \geq C n^{-\frac{1}{2}(d-2)}$. This is a consequence of a Carleman inequality proved in Lemma 3.1 of [6] - see [9] for further discussion.

4) Note that the functions in Lemma 1.1 as well as in Theorem 1 are real valued. It is slightly easier to obtain complex valued examples, but we regarded this as cheating, for several reasons, especially the fact that topological issues are in principle relevant when one wants a lower bound on the gradient such as (iv). On the other hand, all the positive results mentioned above including the one in remark 3) are proved using versions of the Carleman method and are therefore valid also for complex valued or for that matter vector valued functions (Added in proof: the two dimensional result in [4,Theorem 3] should be excepted here).

The proof of Lemma 1.1 is based on considerations involving certain explicit functions $w_{n}$ which we now define. Assume $d \geq 3$ and let

$$
Q_{n}(x)=\int_{-\pi}^{\pi}\left(\left|\bar{x}-e^{i \phi}\right|^{2}+|\overline{\bar{x}}|^{2}\right)^{-\left(\frac{d-2}{2}\right)} e^{i n \phi} d \phi
$$

where we are identifying $\bar{x}$ with $x_{1}+i x_{2}$ as previously discussed. Up to a multiplicative constant $Q_{n}$ is the potential of the measure $e^{i n \theta} d \theta$, so is harmonic except on $\{x: r=1, \rho=0\}$ and vanishes at infinity. $Q_{n}$ obeys the following symmetries:

$$
\begin{gathered}
Q_{n}\left(e^{i \theta} \bar{x}, \overline{\bar{x}}\right)=e^{i n \theta} Q_{n}(\bar{x}, \overline{\bar{x}}) \\
Q_{n}(\bar{x}, \overline{\bar{x}})=Q_{n}(\bar{x}, \overline{\bar{y}}) \quad \text { if } \quad|\overline{\bar{x}}|=|\overline{\bar{y}}| .
\end{gathered}
$$

It follows that $Q_{n}$ has the form

$$
Q_{n}(x)=d_{n}(r, \rho) r^{n} e^{i n \theta}
$$


The function $d_{n}$ is real since the definition (1) shows $Q_{n}$ is real on the $x_{1}$ axis. Let $q_{n}$ be the degree $n$ Taylor polynomial of $Q_{n}$ at the origin. The symmetry (2) is valid for $q_{n}$ and it follows that $q_{n}$ has the form

$$
q_{n}(x)=c_{n}^{(d)} r^{n} e^{i n \theta}
$$

where $c_{n}^{(d)}$ is a real constant. (To see this, write out $q_{n}$ in the form

$$
\sum_{j+k \leq n} p_{j k}(\overline{\bar{x}})\left(r e^{i \theta}\right)^{j}\left(r e^{-i \theta}\right)^{k},
$$

and then compare coefficients of powers of $e^{i \theta}$ in 2). We will show below that $c_{n}^{(d)} \neq 0$.

Let $\phi$ be a nonnegative $C_{0}^{\infty}$ function on $\mathbb{R}$ with support in $(-1,1)$ and with $\int \phi=1$ and (for a certain $\epsilon>0$ to be determined later) let $\phi_{\epsilon}(s)=\epsilon^{-1} \phi\left(\frac{s-1}{\epsilon}\right)$. Define $w_{n}: \mathbb{R}^{d} \backslash\{x: 1-\epsilon \leq r \leq 1+\epsilon, \rho=0\} \rightarrow \mathbb{C}$ by

$$
w_{n}(x)=\left(c_{n}^{(d)}\right)^{-1} \int \phi_{\epsilon}(s) s^{n}\left(Q_{n}\left(\frac{x}{s}\right)-q_{n}\left(\frac{x}{s}\right)\right) d s .
$$

The properties we need for $w_{n}$ are given in the next lemma.

Lemma 1.2. Suppose that $\delta>0$ is given. Then provided $\epsilon>0$ is sufficiently small and $n>n(\delta, \epsilon)$ is sufficiently large, the function $w_{n}$ will have the following properties: $w_{n}$ is even if $n$ is even and odd if $n$ is odd and

(i) $w_{n}=\mathcal{O}\left(|x|^{n}\right)$ at $\infty, w_{n}=\mathcal{O}\left(|x|^{n+2}\right)$ at 0 .

(ii) $\triangle w_{n}=0$.

(iii) $w_{n}(x)=a_{n}(r, \rho) r^{n} e^{i n \theta}$ where $a_{n}$ satisfies: let $R_{n}=\{1-4 \epsilon \leq r \leq$ $\left.1+4 \epsilon, \rho \leq n^{-1 / 2}\right\} \backslash\left\{1-2 \epsilon \leq r \leq 1+2 \epsilon, \rho \leq\left(\frac{\delta}{n}\right)^{1 / 2}\right\}$. Then

$$
\begin{gathered}
\left|a_{n}\right| \leq C(\delta, \epsilon), \quad \text { if } x \in R_{n}, \\
\left|\frac{d a_{n}}{d r}\right|+n^{-1 / 2}\left|\frac{d a_{n}}{d \rho}\right| \leq C(\delta, \epsilon), \quad x \in R_{n}, \\
\frac{1}{2} \leq\left|a_{n}\right| \leq 2, \quad \text { if } x \in R_{n} \text { and } \rho>\left(\frac{\delta}{n}\right)^{1 / 2} .
\end{gathered}
$$

Remark. The set $R_{n}$ is of course a rectangle containing the singularities of $w_{n}$ with a smaller such rectangle deleted. The estimates (7)-(9) say that as long as one stays away from the singularities, $a_{n}$ will be a slowly varying "amplitude" function. Furthermore, as indicated by (9) $a_{n}$ will be bounded 
away from zero and actually will be a small perturbation of the constant function -1 provided $\rho$ is kept bounded below by a constant multiple of $n^{-\frac{1}{2}}$. These properties give a lower bound on $\left|\nabla w_{n}\right|$, since $\left|\nabla\left(r^{n} e^{i n \theta}\right)\right|$ is of course large. This will eventually imply (iv) of Lemma 1.1 for the function $u_{n}$ defined by (19) below.

In fact, it is clear from the definitions that

$$
a_{n}(r, \rho)=\left(c_{n}^{(d)}\right)^{-1} \int \phi_{\epsilon}(s) d_{n}\left(\frac{r}{s}, \frac{\rho}{s}\right) d s-1
$$

and $d_{n}(r, \rho)$ will turn out to be small when $\rho$ is on the order of $n^{-\frac{1}{2}}$. This will follow from Lemma 1.6(i) and the bound for $\left(\frac{\omega}{r}\right)^{n}$ given by Lemma 1.5(i).

Proof of Lemma 1.2. The symmetries (2) and (3) are clearly also valid for $w_{n}$ and imply that $w_{n}$ is even if $n$ is even and odd if $n$ is odd, since $\theta_{-x}=\theta_{x}+\pi$. Next $Q_{n}-q_{n}$ is $\mathcal{O}\left(|x|^{n}\right)$ at $\infty$ since $Q_{n}$ is bounded and $q_{n}$ is a polynomial of degree $n$. Consequently $w_{n}$ is $\mathcal{O}\left(|x|^{n}\right)$ at $\infty$. $Q_{n}-q_{n}$ is $\mathcal{O}\left(|x|^{n+1}\right)$ at 0 by Taylor's theorem and therefore $w_{n}$ is $\mathcal{O}\left(|x|^{n+1}\right)$ at 0 . But $w_{n}$ is even or odd depending on $\mathrm{n}$ and it follows that there are no degree $n+1$ terms in its Taylor expansion. So $w_{n}$ is $\mathcal{O}\left(|x|^{n+2}\right)$ at 0 . This proves (i). To prove (ii), observe that $q_{n}$ is harmonic since $Q_{n}$ is harmonic near 0 (or by (4)). It follows that $Q_{n}-q_{n}$ is harmonic except on $r=1, \rho=0$ and therefore $w_{n}$ is harmonic on its domain $\mathbb{R}^{d} \backslash\{1-\epsilon \leq r \leq 1+\epsilon, \rho=0\}$. It is clear from (4), (5), (6) that $w_{n}$ has the form $a_{n}(r, \rho) r^{n} e^{i n \theta}$, so we only need to show that $c_{n}^{(d)} \neq 0$ and to prove the estimates $(7)-(9)$.

We fix $x \in \mathbb{R}^{d}$, with $\theta=0$ and $r \neq 0,(r, \rho) \neq(1,0)$. Then $\frac{1+r^{2}+\rho^{2}}{r}>2$ so the equation

$$
t^{2}-\frac{1+r^{2}+\rho^{2}}{r} t+1=0
$$

has two roots $\omega$ and $\omega^{-1}$ with $\omega \in(0,1)$. Our calculations will be based on the following contour integration formula.

Lemma 1.3. If $\theta=0, r \neq 0,(r, \rho) \neq(1,0)$ then

$$
Q_{n}(x)=\int_{\gamma_{1}}\left(\zeta^{-1}(\zeta-1)\right)^{-\left(\frac{d-2}{2}\right)}\left(\omega^{-1}-\omega \zeta\right)^{-\left(\frac{d-2}{2}\right)} \zeta^{n} \frac{d \zeta}{i \zeta} r^{-\left(\frac{d-2}{2}\right)} \omega^{n}
$$

for any contour $\gamma_{1} \subset D\left(0, \omega^{-2}\right) \backslash[0,1]$ with $\operatorname{ind}\left(\gamma_{1}, 1\right)=1$. 
Remark. The function $\left(\zeta^{-1}(\zeta-1)\right)^{-\left(\frac{d-2}{2}\right)}$ has a single valued branch on $\mathbb{C} \backslash[0,1]$ and the function $\left(\omega^{-1}-\omega \zeta\right)^{-\left(\frac{d-2}{2}\right)}$ has a single valued branch on $D\left(0, \omega^{-2}\right)$. We take the branches which are positive when $\zeta=-1$.

Proof of Lemma 1.3. We have

$$
\begin{aligned}
Q_{n}(x) & =\int_{-\pi}^{\pi}\left(\left|r-e^{i \phi}\right|^{2}+\rho^{2}\right)^{-\left(\frac{d-2}{2}\right)} e^{i n \phi} d \phi \\
& =\int_{|\zeta|=1}\left((\zeta-r)\left(\zeta^{-1}-r\right)+\rho^{2}\right)^{-\left(\frac{d-2}{2}\right)} \zeta^{n} \frac{d \zeta}{i \zeta} .
\end{aligned}
$$

Also $(\zeta-r)\left(\zeta^{-1}-r\right)+\rho^{2}=-r \zeta^{-1}\left(\zeta^{2}-\frac{1+r^{2}+\rho^{2}}{r}+1\right)=-r \zeta^{-1}(\zeta-\omega)\left(\zeta-\omega^{-1}\right)$, so in view of the remark before the proof we can write

$$
Q_{n}(x)=\int_{|\zeta|=1}\left(\zeta^{-1}(\zeta-\omega)\right)^{-\left(\frac{d-2}{2}\right)}\left(\omega^{-1}-\zeta\right)^{-\left(\frac{d-2}{2}\right)} \zeta^{n} \frac{d \zeta}{i \zeta} r^{-\left(\frac{d-2}{2}\right)}
$$

By change of variables $\zeta \rightarrow \omega \zeta$

$$
Q_{n}(x)=\int_{|\zeta|=\omega^{-1}}\left(\zeta^{-1}(\zeta-1)\right)^{-\left(\frac{d-2}{2}\right)}\left(\omega^{-1}-\omega \zeta\right)^{-\left(\frac{d-2}{2}\right)} \zeta^{n} \frac{d \zeta}{i \zeta} r^{-\left(\frac{d-2}{2}\right)} \omega^{n} .
$$

and the lemma now follows by Cauchy's theorem.

Lemma 1.4. If $n$ is sufficiently large then $\left|c_{n}^{(d)}\right| \approx n^{\left(\frac{d}{2}-2\right)}$. In particular $c_{n}^{(d)} \neq$ 0 .

Proof. Clearly $c_{n}^{(d)}=\lim _{r \rightarrow 0} r^{-n} Q_{n}(r, 0, \ldots, 0)$. Since $\omega(r, 0)=r$ we have

$$
\begin{aligned}
c_{n}^{(d)} & =\lim _{r \rightarrow 0} \int_{\gamma_{1}}\left(\zeta^{-1}(\zeta-1)\right)^{-\left(\frac{d-2}{2}\right)}\left(1-r^{2}\right)^{-\left(\frac{d-2}{2}\right)} \zeta^{n} \frac{d \zeta}{i \zeta} \\
& =\int_{\gamma_{1}}\left(\zeta^{-1}(\zeta-1)\right)^{-\left(\frac{d-2}{2}\right)} \zeta^{n} \frac{d \zeta}{i \zeta} .
\end{aligned}
$$

If $d=3$, the integral can be moved onto the segment [0,1], i.e.

$$
c_{n}^{(3)}=2 \int_{0}^{1}\left(x^{-1}(1-x)\right)^{-1 / 2} x^{n} \frac{d x}{x} .
$$

This easily implies $\left|c_{n}^{(3)}\right| \approx n^{-1 / 2}$ as claimed. Also $c_{n}^{(4)}$ can be evaluated using the residue theorem at the pole -1 , leading to $c_{n}^{(4)}=2 \pi$ for all $\mathrm{n}$, again as claimed. When $d \geq 5$ the integral is less easy to work with, so we proceed as follows: denoting $Q_{n}(r, 0, \ldots, 0)$ by $T_{n}^{(d)}(r)$, the definition (1) implies

$$
\frac{d T_{n}^{(d)}}{d r}=(2-d)\left(r T_{n}^{d+2}-\frac{1}{2} T_{n-1}^{(d+2)}-\frac{1}{2} T_{n+1}^{(d+2)}\right)
$$


Comparing terms of degree $n-1$ in the Taylor expansion at 0 gives

$$
n c_{n}^{(d)}=(2-d)\left(-\frac{1}{2} c_{n-1}^{(d+2)}\right)
$$

and therefore also

$$
c_{n}^{(d)}=\left(\frac{d}{2}-1\right)^{-1}(n+1) c_{n+1}^{(d-2)} .
$$

The lemma now follows from the three and four dimensional cases by induction on $\mathrm{d}$.

We want to use Lemma 1.3 to do asymptotics for $Q_{n}$. We need some bounds for $\omega$, which we formulate as a lemma.

Lemma 1.5. Assume $\frac{1}{2} \leq r \leq 2, \rho \leq 1$. Then

(i) $\omega \leq \min \left(r, r^{-1}\right)$ and $\min \left(r, r^{-1}\right)-\omega \approx \frac{\rho^{2}}{|1-r|+\rho}$.

(ii) $\omega^{-1}-\omega \approx \rho+|1-r|$.

(iii) $\left|\frac{\partial}{\partial r}\left(\frac{\omega}{r}\right)\right| \lesssim 1$.

(iv) $\left|\frac{\partial}{\partial r}\left(\frac{\omega}{r}\right)\right| \lesssim\left(\frac{\rho}{|1-r|+\rho}\right)^{2}$ when $r<1$.

(v) $\left|\frac{\partial}{\partial \rho}\left(\frac{\omega}{r}\right)\right| \lesssim \frac{\rho}{|1-r|+\rho}$.

Proof. (i) By the quadratic formula

$$
\omega=\frac{1+r^{2}+\rho^{2}-\sqrt{\left(1+r^{2}+\rho^{2}\right)^{2}-4 r^{2}}}{2 r}
$$

which may be rewritten as

$$
\begin{aligned}
& \omega=\frac{1+r^{2}+\rho^{2}-\left(\left|1-r^{2}\right|+\rho^{2}\right)}{2 r} \\
& \quad-\frac{\left|1-r^{2}\right|+\rho^{2}}{2 r}\left(\sqrt{1+\frac{2 \rho^{2}\left(1+r^{2}-\left|1-r^{2}\right|\right)}{\left(\left|1-r^{2}\right|+\rho^{2}\right)^{2}}}-1\right) .
\end{aligned}
$$

The first term on the right side is $\min \left(r, \frac{1}{r}\right)$. Estimating the second term using $\sqrt{1+x}-1 \approx \min (x, \sqrt{x})$ gives

$$
\min \left(r, \frac{1}{r}\right)-\omega \approx \frac{\left|1-r^{2}\right|+\rho^{2}}{2 r} \min (x, \sqrt{x})
$$

where $x=\frac{2 \rho^{2}\left(1+r^{2}-\left|1-r^{2}\right|\right)}{\left(\left|1-r^{2}\right|+\rho^{2}\right)^{2}}$. The assumption on $r$ implies $2 r \approx 1,1+r^{2}-\mid 1-$ $r^{2}|\approx 1| 1-,r^{2}|\approx| 1-r \mid$, so

$$
\min \left(r, \frac{1}{r}\right)-\omega \approx \min \left(\rho, \frac{\rho^{2}}{|1-r|+\rho^{2}}\right) .
$$


Considering cases $(\rho \leq|1-r|, \rho \geq|1-r|)$ we see that this implies (i).

(ii) We record the fact that

$$
|1-r|+\frac{\rho^{2}}{|1-r|+\rho} \approx|1-r|+\rho
$$

which follows by considering cases $|1-r| \leq \rho,|1-r| \geq \rho$. Since $\omega \approx 1$, (i) implies $\omega^{-1}-\omega \approx 1-\omega \approx|1-r|+\frac{\rho^{2}}{|1-r|+\rho}$.

(iii) and (iv) Differentiate equation (10) for $r$ obtaining

$$
\left(2 \omega-\frac{1+r^{2}+\rho^{2}}{r}\right) \frac{\partial \omega}{\partial r}=\omega \frac{\partial}{\partial r}\left(\frac{1+r^{2}+\rho^{2}}{r}\right) .
$$

Part (i) implies that

$$
\begin{aligned}
\frac{1+r^{2}+\rho^{2}}{r}-2 \omega & =\frac{1+r^{2}}{r}-2 \min \left(r, \frac{1}{r}\right)+E \\
& =\left|\frac{1}{r}-r\right|+E
\end{aligned}
$$

with $E \approx \frac{\rho^{2}}{|1-r|+\rho}$. Therefore

$$
\begin{aligned}
\omega^{-1} \frac{\partial \omega}{\partial r}-\frac{1}{r} & =\frac{\frac{1}{r}-r-\left|\frac{1}{r}-r\right|+\frac{\rho^{2}}{r}-E}{r\left(\left|\frac{1}{r}-r\right|+E\right)} \\
& \approx \frac{\frac{1}{r}-r-\left|\frac{1}{r}-r\right|+\frac{\rho^{2}}{r}-E}{|1-r|+\rho}
\end{aligned}
$$

by (12). If $r<1$ it follows that

$$
\left|\omega^{-1} \frac{\partial \omega}{\partial r}-\frac{1}{r}\right| \leq \frac{\rho^{2}+E}{|1-r|+\rho} \lesssim \frac{\rho^{2}}{(|1-r|+\rho)^{2}}
$$

by the bounds on $E$. This implies (iv) since $\left|\frac{\partial}{\partial r}\left(\frac{\omega}{r}\right)\right|=\frac{\omega}{r}\left|\omega^{-1} \frac{\partial \omega}{\partial r}-\frac{1}{r}\right|$. If $r>1$ we have instead

$$
\left|\omega^{-1} \frac{\partial \omega}{\partial r}-\frac{1}{r}\right| \lesssim 1+\frac{\rho^{2}}{(|1-r|+\rho)^{2}} \lesssim 1
$$

which implies (iii).

(v) Differentiate (10) for $\rho$ obtaining

$$
\begin{gathered}
\left(2 \omega-\frac{1+r^{2}+\rho^{2}}{r}\right) \frac{d \omega}{d \rho}=\omega \frac{d}{d \rho} \frac{1+r^{2}+\rho^{2}}{r} \\
\omega^{-1} \frac{d \omega}{d \rho}=\frac{2 \rho / r}{\left|\frac{1}{r}-r\right|+E} \lesssim \frac{\rho}{|1-r|+\rho}
\end{gathered}
$$


by (12). This implies (v).

Lemma 1.6. With $d_{n}$ defined by (4) we have

(i) $\left|d_{n}(r, \rho)-c_{n}^{(d)} r^{-\left(\frac{d-2}{2}\right)}\left(\omega^{-1}-\omega\right)^{-\left(\frac{d-2}{2}\right)}\left(\frac{\omega}{r}\right)^{n}\right| \lesssim n^{\frac{d}{2}-3}\left(\omega^{-1}-\omega\right)^{-\frac{d}{2}}\left(\frac{\omega}{r}\right)^{n}$

(ii) $\left|\frac{\partial d_{n}}{\partial r}\right| \lesssim n^{\frac{d}{2}-2}\left(\frac{\omega}{r}\right)^{n}\left(\omega^{-1}-\omega\right)^{-\frac{d}{2}}+n^{\frac{d}{2}-1}\left(\frac{\omega}{r}\right)^{n}\left(\omega^{-1}-\omega\right)^{-\left(\frac{d-2}{2}\right)}\left|\frac{\partial\left(\frac{\omega}{r}\right)}{\partial r}\right|$

(iii) $\left|\frac{\partial d_{n}}{\partial \rho}\right| \lesssim n^{\frac{d}{2}-1}\left(\frac{\omega}{r}\right)^{n}\left(\omega^{-1}-\omega\right)^{-\left(\frac{d-2}{2}\right)}\left|\frac{\partial\left(\frac{\omega}{r}\right)}{\partial \rho}\right|$

provided $\frac{1}{2} \leq r \leq 2, \rho \leq 1$ and $|1-r|+\rho \geq \frac{1}{n}$.

Proof. Lemma 1.3 implies that

$$
d_{n}(r, \rho)=r^{-\left(\frac{d-2}{2}\right)}\left(\frac{\omega}{r}\right)^{n} \int_{\gamma_{1}}\left(\zeta^{-1}(\zeta-1)\right)^{-\left(\frac{d-2}{2}\right)}\left(\omega^{-1}-\omega \zeta\right)^{-\left(\frac{d-2}{2}\right)} \zeta^{n} \frac{d \zeta}{i \zeta}
$$

where we take $\gamma_{1}$ to be the positively oriented rectangle with vertices at $\frac{-b}{n}-i \frac{b}{n}$, $1+\frac{b}{n}-i \frac{b}{n}, 1+\frac{b}{n}+i \frac{b}{n}, \frac{-b}{n}+i \frac{b}{n}$, with b being a small positive constant. Using (11) to evaluate $c_{n}^{(d)}$ we obtain

$$
\begin{aligned}
\left|d_{n}(r, \rho)-c_{n}^{(d)} r^{-\left(\frac{d-2}{2}\right)}\left(\omega^{-1}-\omega\right)^{-\left(\frac{d-2}{2}\right)}\left(\frac{\omega}{r}\right)^{n}\right| & \\
\lesssim \int_{\gamma_{1}}\left|\zeta^{-1}(\zeta-1)\right|^{-\left(\frac{d-2}{2}\right)} \mid\left(\omega^{-1}-\omega \zeta\right)^{-\left(\frac{d-2}{2}\right)}- & \left(\omega^{-1}-\omega\right)^{-\left(\frac{d-2}{2}\right)} \mid \\
& \times|\zeta|^{n} \frac{|d \zeta|}{|\zeta|} r^{-\left(\frac{d-2}{2}\right)}\left(\frac{\omega}{r}\right)^{n}
\end{aligned}
$$

Here

$$
\left|\left(\omega^{-1}-\omega \zeta\right)^{-\left(\frac{d-2}{2}\right)}-\left(\omega^{-1}-\omega\right)^{-\left(\frac{d-2}{2}\right)}\right| \lesssim|\zeta-1| \max _{z \in \overline{\zeta 1}}\left|\omega^{-1}-\omega z\right|^{-\frac{d}{2}}
$$

by the mean value theorem. The segment $\overline{\zeta 1}$ is inside the rectangle $\gamma_{1}$, which is contained in $D\left(0,1+\frac{\sqrt{2} b}{n}\right)$, hence in $D\left(0, \omega^{-1}\right)$ if $\mathrm{b}$ is small, by assumption and (ii) of Lemma 1.5. We conclude that

$$
\begin{aligned}
\left|\left(\omega^{-1}-\omega \zeta\right)^{-\left(\frac{d-2}{2}\right)}-\left(\omega^{-1}-\omega\right)^{-\left(\frac{d-2}{2}\right)}\right| & \lesssim|\zeta-1|\left(\omega^{-1}-1\right)^{-\frac{d}{2}} \\
& \approx|\zeta-1|\left(\omega^{-1}-\omega\right)^{-\frac{d}{2}}
\end{aligned}
$$

Therefore

$$
\begin{aligned}
& \left|d_{n}(r, \rho)-c_{n}^{(d)} r^{-\left(\frac{d-2}{2}\right)}\left(\omega^{-1}-\omega\right)^{-\left(\frac{d-2}{2}\right)}\left(\frac{\omega}{r}\right)^{n}\right| \\
\lesssim & \int_{\gamma_{1}}\left|\zeta^{-1}(\zeta-1)\right|^{-\left(\frac{d-2}{2}\right)}|\zeta-1||\zeta|^{n} \frac{|d \zeta|}{|\zeta|}\left(\omega^{-1}-\omega\right)^{-\frac{d}{2}} r^{-\left(\frac{d-2}{2}\right)}\left(\frac{\omega}{r}\right)^{n}
\end{aligned}
$$


The integral is easily seen to be $\lesssim n^{\frac{d}{2}-3}$ ( the main contribution is when $|\zeta| \geq 1-\frac{1}{n}$ ) and (i) follows.

Note that (i) implies

$$
\left|d_{n}\right| \lesssim n^{\frac{d}{2}-2}\left(\omega^{-1}-\omega\right)^{-\left(\frac{d-2}{2}\right)}\left(\frac{\omega}{r}\right)^{n}
$$

since $\left|c_{n}^{(d)}\right| \approx n^{\frac{d}{2}-2}$ and the right side of (i) is lower order since $\omega^{-1}-\omega \gtrsim \frac{1}{n}$ by Lemma 1.5 (ii). The right side of (ii) dominates the right side of (14), so by the product rule it suffices to prove (ii) with $d_{n}$ replaced by $r^{\frac{d}{2}-1} d_{n}$ on the left hand side. With the same $\gamma_{1}$ as before we have

$$
\begin{aligned}
& \frac{\partial}{\partial r}\left(r^{\frac{d-2}{2}} d_{n}\right) \\
& =\left(\frac{\omega}{r}\right)^{n} \int_{\gamma_{1}}\left(\zeta^{-1}(\zeta-1)\right)^{-\left(\frac{d-2}{2}\right)} \frac{\partial}{\partial r}\left(\omega^{-1}-\omega \zeta\right)^{-\left(\frac{d-2}{2}\right)} \zeta^{n} \frac{d \zeta}{i \zeta} \\
& \quad+n\left(\frac{\omega}{r}\right)^{n-1} \frac{\partial}{\partial r}\left(\frac{\omega}{r}\right) \int_{\gamma_{1}}\left(\zeta^{-1}(\zeta-1)\right)^{-\left(\frac{d-2}{2}\right)}\left(\omega^{-1}-\omega \zeta\right)^{-\left(\frac{d-2}{2}\right)} \zeta^{n} \frac{d \zeta}{i \zeta}
\end{aligned}
$$

Here $\left|\frac{\partial}{\partial r}\left(\omega^{-1}-\omega \zeta\right)^{-\left(\frac{d-2}{2}\right.}\right| \lesssim\left|\frac{\partial \omega}{\partial r}\right|\left|\omega^{-1}-\omega \zeta\right|^{-\frac{d l}{2}}$ which is $\lesssim\left|\frac{\partial \omega}{\partial r}\right|\left(\omega^{-1}-\omega\right)^{-\frac{l l}{2}}$, as above. Using (iii) of Lemma 1.5 we may bound the first term on the right side of (15) by

$$
\left(\frac{\omega}{r}\right)^{n}\left(\omega^{-1}-\omega\right)^{-\frac{d}{2}} \int_{\gamma_{1}}\left|\zeta^{-1}(\zeta-1)\right|^{-\left(\frac{d-2}{2}\right)}|\zeta|^{n} \frac{|d \zeta|}{|\zeta|} \lesssim n^{\frac{d}{2}-2}\left(\frac{\omega}{r}\right)^{n}\left(\omega^{-1}-\omega\right)^{-\frac{d}{2}} .
$$

The second term is similarly $\lesssim n^{\frac{d}{2}-1}\left(\frac{\omega}{r}\right)^{n}\left(\omega^{-1}-\omega\right)^{-\left(\frac{d-2}{2}\right)}\left|\frac{\partial}{\partial r}\left(\frac{\omega}{r}\right)\right|$ and (ii) follows. (iii) may be done essentially the same: taking the $\rho$ derivative of (13), then putting absolute values inside leads to

$\left|\frac{\partial d_{n}}{\partial \rho}\right| \lesssim n^{\frac{d}{2}-2}\left(\frac{\omega}{r}\right)^{n}\left(\omega^{-1}-\omega\right)^{-\frac{d}{2}}\left|\frac{\partial}{\partial \rho}\left(\frac{\omega}{r}\right)\right|+n^{\frac{d l}{2}-1}\left(\frac{\omega}{r}\right)^{n}\left(\omega^{-1}-\omega\right)^{-\left(\frac{d-2}{2}\right)}\left|\frac{\partial}{\partial \rho}\left(\frac{\omega}{r}\right)\right|$.

Lemma 1.5 (ii) implies that the second term on the right hand side dominates the first and (iii) follows.

Completion of proof of Lemma 1.2. We have (from the definitions)

$$
a_{n}(r, \rho)=\left(c_{n}^{d}\right)^{-1} \int \phi_{\epsilon}(s) d_{n}\left(\frac{r}{s}, \frac{\rho}{s}\right) d s-1 .
$$

We assume $(r, \rho)$ belongs to $R_{n}$ and $\epsilon$ is small and $\mathrm{n}$ large. Then $\left(\frac{r}{s}, \frac{\rho}{s}\right)$ will satisfy the hypotheses of Lemmas 1.5 and 1.6, e.g. $\frac{r}{s} \in\left(\frac{1}{2}, 2\right)$. Formula 
(14) and Lemma 1.4 imply that $\left|d_{n}\left(\frac{r}{s}, \frac{\rho}{s}\right)\right| \lesssim\left|c_{n}^{(d)}\right|\left(\omega^{-1}-\omega\right)^{-\left(\frac{d-2}{2}\right)}\left(\frac{s \omega}{r}\right)^{n}$, where $\omega=\omega\left(\frac{r}{s}, \frac{\rho}{s}\right)$. Therefore

$$
\left|1+a_{n}(r, \rho)\right| \lesssim \int \phi_{\epsilon}(s)\left(\omega^{-1}-\omega\right)^{-\left(\frac{d-2}{2}\right.}\left(\frac{s \omega}{r}\right)^{n} d s
$$

We record the fact that

$$
\left(\frac{s \omega}{r}\right)^{n} \leq C_{2} \min \left(1, \frac{s}{r}\right)^{2 n} e^{-C_{1} \frac{|s-r|+n \rho}{|s-r|+\rho}}
$$

when $(r, \rho) \in R_{n}$ and $1-\epsilon \leq s \leq 1+\epsilon$, for certain constants $C_{1}$ and $C_{2}$. Inequality (17) is proved as follows: Lemma 1.5 (i) implies

$$
\begin{aligned}
\frac{s \omega}{r} & \leq \min \left(1,\left(\frac{s}{r}\right)^{2}\right)-C_{0} \frac{\rho^{2}}{|s-r|+\rho} \\
& \leq \min \left(1,\left(\frac{s}{r}\right)^{2}\right)\left(1-\frac{C_{0}}{4} \frac{\rho^{2}}{|s-r|+\rho}\right)
\end{aligned}
$$

where the last line uses $\frac{s}{r} \leq 2$. Consequently

$$
\left(\frac{s \omega}{r}\right)^{n} \leq \min \left(1, \frac{s}{r}\right)^{2 n} e^{-\frac{C_{0}}{4} \frac{n \rho^{2}}{|s-r|+\rho}}
$$

and (17) follows with $C_{1}=\frac{C_{0}}{4}, C_{2}=e^{C_{1}}$.

Define $E_{k}: \mathbb{R}^{+} \rightarrow \mathbb{R}$ by $E_{k}(x)=x^{\frac{k}{2}} e^{-C_{1} x}$. Lemma 1.5 (ii) implies that $\omega^{-1}-\omega \approx|s-r|+\rho$. So by $(17)$

$$
\begin{aligned}
\left|1+a_{n}(r, \rho)\right| & \lesssim \int \phi_{\epsilon}(s)(|s-r|+\rho)^{-\left(\frac{d-2}{2}\right)} e^{-C_{1} \frac{|s-r|+n \rho^{2}}{|s-r|+\rho}} d s \\
& =\int \phi_{\epsilon}(s)\left(|s-r|+n \rho^{2}\right)^{-\left(\frac{d-2}{2}\right)} E_{d-2}\left(\frac{|s-r|+n \rho^{2}}{|s-r|+\rho}\right) d s .
\end{aligned}
$$

We now prove (7). Namely, if $(r, \rho) \in R_{n}, 1-\epsilon \leq s \leq 1+\epsilon$, then $|s-r|+n \rho^{2} \gtrsim$ $\min (\delta, \epsilon)$. Therefore

$$
\left|1+a_{n}(r, \rho)\right| \leq C(\delta, \epsilon) \int \phi_{\epsilon}(s) E_{d-2}\left(\frac{|s-r|+n \rho^{2}}{|s-r|+\rho}\right) d s .
$$

$E_{d-2}$ is of course a bounded function, so (7) is proved. Next we prove (9). If $\rho \geq \sqrt{\frac{\delta}{n}}$ then by $(18)$

$$
\begin{aligned}
\left|1+a_{n}(r, \rho)\right| & \leq C(\delta) \int \phi_{\epsilon}(s) E_{d-2}\left(\frac{|s-r|+n \rho^{2}}{|s-r|+\rho}\right) d s \\
& \leq C(\delta) \max _{1-\epsilon \leq s \leq 1+\epsilon} E_{d-2}\left(\frac{|s-r|+n \rho^{2}}{|s-r|+\rho}\right) .
\end{aligned}
$$


Of course $E_{d-2}(x) \rightarrow 0$ as $x \rightarrow+\infty$. On the other hand, if $A$ is any preassigned number then by taking $\epsilon$ small enough and $n$ large enough we can insure that $\frac{|s-r|+n \rho^{2}}{|s-r|+\rho} \geq A$ for all $r \in(1-4 \epsilon, 1+4 \epsilon), \rho \geq \sqrt{\frac{\delta}{n}}$ and $s \in(1-\epsilon, 1+\epsilon)$ If we do this with $A=\frac{1}{2 C(\delta)}$ we have proved (9).

It remains to prove the derivative estimates (8). We first consider $\frac{\partial a_{n}}{\partial \rho}$. Differentiating (16),

$$
\frac{\partial a_{n}}{\partial \rho}=\left(c_{n}^{(d)}\right)^{-1} \int \phi_{\epsilon}(s) \frac{\partial d_{n}}{\partial \rho}\left(\frac{r}{s}, \frac{\rho}{s}\right) \frac{d s}{s} .
$$

By Lemma 1.6(iii) and Lemma 1.4,

$$
\left|\frac{\partial a_{n}}{\partial \rho}\right| \lesssim n \int \phi_{\epsilon}(s)\left(\frac{s \omega}{r}\right)^{n}\left(\omega^{-1}-\omega\right)^{-\left(\frac{d-2}{2}\right)} \lambda(s) d s
$$

where

$$
\lambda(s)=\left|\frac{\partial\left(\frac{\omega}{r}\right)}{\partial \rho}\left(\frac{r}{s}, \frac{\rho}{s}\right)\right| \lesssim \frac{\rho}{|s-r|+\rho}
$$

by Lemma $1.5(\mathrm{v})$ and (ii). So

$$
\begin{aligned}
\left|\frac{\partial a_{n}}{\partial \rho}\right| & \lesssim n \rho \int \phi_{\epsilon}(s)\left(\frac{s \omega}{r}\right)^{n}(|s-r|+\rho)^{-\frac{d}{2}} d s \\
& \lesssim n \rho \int \phi_{\epsilon}(s)\left(|s-r|+n \rho^{2}\right)^{-\frac{d}{2}} E_{d}\left(\frac{|s-r|+n \rho^{2}}{|s-r|+\rho}\right) d s \\
& \lesssim C(\delta, \epsilon) n \rho .
\end{aligned}
$$

In the next to last inequality we used (17) in the same way as in the proof of (7). Since $\rho \leq n^{-\frac{1}{2}}$ on $R_{n}$ it follows that $n^{\frac{1}{2}}\left|\frac{\partial a_{n}}{\partial \rho}\right| \leq C(\delta, \epsilon)$ as claimed in (8).

We now prove the bound for $\frac{\partial a_{n}}{\partial r}$ in (8). We differentiate (16) for $\mathrm{r}$ :

$$
\frac{\partial a_{n}}{\partial r}=\left(c_{n}^{(d)}\right)^{-1} \int \phi_{\epsilon}(s) \frac{\partial d_{n}}{\partial r}\left(\frac{r}{s}, \frac{\rho}{s}\right) \frac{d s}{s}
$$

We now proceed as before, estimating $\frac{\partial d_{n}}{\partial r}$ via Lemma 1.6(ii). This gives

$$
\left|\frac{\partial a_{n}}{\partial r}\right| \lesssim \int \phi_{\epsilon}(s)\left(\frac{s \omega}{r}\right)^{n}\left(\omega^{-1}-\omega\right)^{-\frac{d}{2}} d s+n \int \phi_{\epsilon}(s)\left(\frac{s \omega}{r}\right)^{n}\left(\omega^{-1}-\omega\right)^{-\left(\frac{d-2}{2}\right)} \kappa(s) d s .
$$

where

$$
\kappa(s)=\left|\frac{\partial\left(\frac{\omega}{r}\right)}{\partial r}\left(\frac{r}{s}, \frac{\rho}{s}\right)\right| \lesssim \begin{cases}1 & \text { if } s<r \\ \left(\frac{\rho}{|s-r|+\rho}\right)^{2} & \text { if } s \geq r\end{cases}
$$

by Lemma 1.5 (iii), (iv). The first integral may be estimated by $C(\delta, \epsilon)$ just as in the proof of (7): the only difference is the exponent $-\frac{d}{2}$ instead of $-\left(\frac{d-2}{2}\right)$. 
We omit the details of this and will now consider the second integral. We split it into $\int_{s>r}$ and $\int_{s<r}$, and substitute in the bound for $\kappa$ and the bound $\omega^{-1}-\omega \approx|s-r|+\rho$. This gives

$$
\begin{aligned}
\left|\frac{\partial a_{n}}{\partial r}\right| \lesssim C(\delta, \epsilon) & +n \rho^{2} \int_{s>r} \phi_{\epsilon}(s)(|s-r|+\rho)^{-\left(\frac{d+2}{2}\right)}\left(\frac{s \omega}{r}\right)^{n} d s \\
& +n \int_{s<r} \phi_{\epsilon}(s)(|s-r|+\rho)^{-\left(\frac{d-2}{2}\right)}\left(\frac{s \omega}{r}\right)^{n} d s
\end{aligned}
$$

The $s>r$ integral here may be estimated by $C(\delta, \epsilon)$ as before (again the only difference is in the exponent of $|s-r|+\rho)$. We therefore have

$$
\begin{aligned}
\left|\frac{\partial a_{n}}{\partial r}\right| & \lesssim C(\delta, \epsilon)\left(1+n \rho^{2}\right)+n \int_{s<r} \phi_{\epsilon}(s)(|s-r|+\rho)^{-\left(\frac{d-2}{2}\right)}\left(\frac{s \omega}{r}\right)^{n} d s \\
& \leq C(\delta, \epsilon)\left(1+n \rho^{2}\right) \\
& +C n \int_{s<r} \phi_{\epsilon}(s)\left(|s-r|+n \rho^{2}\right)^{-\left(\frac{d-2}{2}\right)} E_{d-2}\left(\frac{|s-r|+n \rho^{2}}{|s-r|+\rho}\right)\left(\frac{s}{r}\right)^{2 n} d s
\end{aligned}
$$

where we used (17). Each of the factors in the integrand is bounded by a constant $C(\delta, \epsilon)$ so we obtain

$$
\left|\frac{\partial a_{n}}{\partial r}\right| \lesssim C(\delta, \epsilon)\left(1+n \rho^{2}+n \int_{s<r}\left(\frac{s}{r}\right)^{2 n} d s\right) .
$$

The integral here is $\lesssim \frac{1}{n}$, so

$$
\left|\frac{\partial a_{n}}{\partial r}\right| \leq C(\delta, \epsilon)\left(1+n \rho^{2}\right)
$$

and now we are done, since by assumption $\rho \leq n^{-\frac{1}{2}}$

Proof of Lemma 1.1. We start by constructing the function $u_{n}$. Let $\gamma$ be a sufficiently small positive constant to be specified later. Let $e^{1}$ and $e^{2}$ be the first two standard basis vectors and define $e_{*}^{1}$ and $e_{*}^{2}$ by

$$
\begin{aligned}
& e_{*}^{1}=\left(1+\frac{\gamma}{n}\right)^{-\frac{1}{2}}\left(1,0,\left(\frac{\gamma}{n}\right)^{\frac{1}{2}}, 0, \ldots, 0\right) \\
& e_{*}^{2}=\left(1+\frac{\gamma}{n}\right)^{-\frac{1}{2}}\left(0,1,0,\left(\frac{\gamma}{n}\right)^{\frac{1}{2}}, 0, \ldots, 0\right) .
\end{aligned}
$$

It is here that we use that $d \geq 4$. For given $x \in \mathbb{R}^{d}$ define coordinates $r_{*}, \theta_{*}, \rho_{*}$ via

$$
x=r_{*} \cos \theta_{*} e_{*}^{1}+r_{*} \sin \theta_{*} e_{*}^{2}+\overline{\bar{x}}_{*}, \rho_{*}=\left|\overline{\bar{x}}_{*}\right|
$$


where $\overline{\bar{x}}_{*} \perp s p\left(e_{*}^{1}, e_{*}^{2}\right)$. In other words $r_{*}, \theta_{*}, \rho_{*}$ are defined like $r, \theta, \rho$ but using the vectors $e_{*}^{1}$ and $e_{*}^{2}$ instead of $e^{1}$ and $e^{2}$. Next let $\delta$ be small enough and choose $\epsilon$ and functions $w_{n}(x)=a_{n}(r, \rho) r^{n} e^{i n \theta}$ by Lemma 1.2. Let $p$ and $q_{n}$ be $C_{0}^{\infty}$ functions on $\mathbb{R}$ with $p(r)=1$ when $|r-1| \leq 2 \epsilon, p(r)=0$ when $|r-1| \geq 3 \epsilon$ and $q_{n}(\rho)=1$ when $\rho \leq \sqrt{\frac{\delta}{n}}, q_{n}(\rho)=0$ when $\rho \geq \sqrt{\frac{2 \delta}{n}}$, and with $\left|\frac{d^{j} p}{d r^{j}}\right| \lesssim \epsilon^{-j},\left|\frac{d^{j} q_{n}}{d \rho^{j}}\right| \leq\left(\frac{\delta}{n}\right)^{-\frac{j}{2}}$. Let $\chi_{n}(r, \rho)=1-p(r) q_{n}(\rho)$ and define

$$
u_{n}(x)=\frac{1}{n}\left(\chi_{n}(r, \rho) a_{n}(r, \rho) r^{n} \cos n \theta+\chi_{n}\left(r_{*}, \rho_{*}\right) a_{n}\left(r_{*}, \rho_{*}\right) r_{*}^{n} \sin n \theta_{*}\right) .
$$

It is clear that $u_{n}$ is even if $\mathrm{n}$ is even and odd if $\mathrm{n}$ is odd and has property (i) of Lemma 1.1. To prove (ii) - (iv) we need the following (elementary) lemma. In parts (iv) and (v), of course we are regarding $\frac{\partial}{\partial r}$ etc. as vector fields.

Lemma 1.7. If $\frac{1}{2} \leq r \leq 2$ and $\rho$ is sufficiently small (independently of $\gamma$ and n) then

(i) $\left|r-r_{*}\right| \leq C\left(\rho \sqrt{\frac{\gamma}{n}}+\frac{\gamma}{n}\right)$.

(ii) $\left|\theta-\theta_{*}\right| \leq C\left(\rho \sqrt{\frac{\gamma}{n}}+\frac{\gamma}{n}\right)$.

(iii) $\left|\rho-\rho_{*}\right| \leq C \sqrt{\frac{\gamma}{n}}$.

(iv) $\left|\frac{\partial}{\partial r}-\frac{\partial}{\partial r_{*}}\right| \leq C \sqrt{\frac{\gamma}{n}}$.

(v) $\left|\frac{1}{r} \frac{\partial}{\partial \theta}-\frac{1}{r_{*}} \frac{\partial}{\partial \theta_{*}}\right| \leq C \sqrt{\frac{\gamma}{n}}$.

Furthermore if $\frac{1}{2} \leq r \leq 2$ then

(vi) $\max \left(\rho, \rho_{*}\right) \geq C^{-1} \sqrt{\frac{\gamma}{n}}$.

Proof. For (i)-(v) the relevant properties of the $e^{i}$ and $e_{*}^{i}$ are that

$$
\begin{gathered}
\left|\left\langle e^{i}-e_{*}^{i}, e^{j}\right\rangle\right| \leq \frac{C \gamma}{n}, i, j \in\{1,2\} \\
\left|e^{i}-e_{*}^{i}\right| \leq C \sqrt{\frac{\gamma}{n}}, i \in\{1,2\}
\end{gathered}
$$

and are easily checked. 
To prove (i) and (ii) we use (20):

$$
\begin{aligned}
\left|r-r_{*}\right| \lesssim\left|r^{2}-r_{*}^{2}\right| & =\left|\sum_{i=1}^{2}\left\langle x, e^{i}\right\rangle^{2}-\left\langle x, e_{*}^{i}\right\rangle^{2}\right| \\
& \leq C \sum_{i=1}^{2}\left|\left\langle x, e^{i}-e_{*}^{i}\right\rangle\right| .
\end{aligned}
$$

Replacing $\mathrm{x}$ here by its projection on the orthogonal complement of $s p\left\{e_{1}, e_{2}\right\}$ introduces an error of $\lesssim \frac{\gamma}{n}$ by $(20)$ and therefore $\left|\left\langle x, e^{i}-e_{*}^{i}\right\rangle\right| \leq C\left(\rho \sqrt{\frac{\gamma}{n}}+\frac{\gamma}{n}\right)$, which gives (i).

To prove (ii) write

$$
\left|\cos \theta-\cos \theta_{*}\right|=\left|\frac{\left\langle x, e^{1}\right\rangle}{r}-\frac{\left\langle x, e_{*}^{1}\right\rangle}{r_{*}}\right| .
$$

For sufficiently small $\rho$ the bound on $\left|r-r_{*}\right|$ implies $r_{*} \geq \frac{1}{4}$ so

$$
\begin{aligned}
\left|\cos \theta-\cos \theta_{*}\right| & \leq C\left|r_{*}\left\langle x, e^{1}\right\rangle-r\left\langle x, e_{*}^{1}\right\rangle\right| \\
& \leq C\left(\left|r-r_{*}\right|\left|\left\langle x, e^{1}\right\rangle\right|+r\left|\left\langle x, e^{1}-e_{*}^{1}\right\rangle\right|\right) \\
& \leq C\left(\left|r-r_{*}\right|+\left|\left\langle x, e^{1}-e_{*}^{1}\right\rangle\right|\right. \\
& \leq C\left(\rho \sqrt{\frac{\gamma}{n}}+\frac{\gamma}{n}\right) .
\end{aligned}
$$

Likewise $\left|\sin \theta-\sin \theta_{*}\right| \leq C\left(\rho \sqrt{\frac{\gamma}{n}}+\frac{\gamma}{n}\right)$ (replace $e^{1}$ and $e_{*}^{1}$ by $e^{2}$ and $e_{*}^{2}$ in the preceding argument) and therefore $\left|\theta-\theta_{*}\right| \leq C\left(\rho \sqrt{\frac{\gamma}{n}}+\frac{\gamma}{n}\right)$, i.e. (ii) holds.

For (iii) we write

$$
\begin{aligned}
\left|\rho-\rho_{*}\right| & =|| x-r \cos \theta e^{1}-r \sin \theta e^{2}|-| x-r_{*} \cos \theta_{*} e_{*}^{1}-r_{*} \sin \theta_{*} e_{*}^{2}|| \\
& \leq\left|r \cos \theta e^{1}-r_{*} \cos \theta_{*} e_{*}^{1}\right|+\left|r \sin \theta e^{2}-r_{*} \sin \theta_{*} e_{*}^{2}\right| .
\end{aligned}
$$

(iii) now follows easily using (i), (ii) and (21) and the triangle inequality.

For (iv) and (v) write

$$
\begin{gathered}
\left|\frac{\partial}{\partial r}-\frac{\partial}{\partial r_{*}}\right|=\left|\cos \theta e^{1}+\sin \theta e^{2}-\cos \theta_{*} e_{*}^{1}-\sin \theta_{*} e_{*}^{2}\right|, \\
\left|\frac{1}{r} \frac{\partial}{\partial \theta}-\frac{1}{r_{*}} \frac{\partial}{\partial \theta_{*}}\right|=\left|-\sin \theta e^{1}+\cos \theta e^{2}+\sin \theta_{*} e_{*}^{1}-\cos \theta_{*} e_{*}^{2}\right| .
\end{gathered}
$$

Now argue as in the proof of (iii). 
We now prove (vi). The definition of $e_{*}^{j}$ shows that

$$
\sum_{j=1}^{2}<x, e_{*}^{j}>^{2} \leq\left(1+\frac{\gamma}{n}\right)^{-1} \sum_{j=1}^{2}<x, e_{j}>^{2}+\left(1+\frac{\gamma}{n}\right)^{-1} \frac{\gamma}{n} \rho^{2} .
$$

Therefore

$$
\begin{aligned}
\rho_{*}^{2} & \geq|x|^{2}-\left(1+\frac{\gamma}{n}\right)^{-1}|x|^{2}-\left(1+\frac{\gamma}{n}\right)^{-1} \frac{\gamma}{n} \rho^{2} \\
& =\frac{\gamma}{n}\left(1+\frac{\gamma}{n}\right)^{-1}\left(|x|^{2}-\rho^{2}\right)
\end{aligned}
$$

so that $\rho \leq \frac{1}{2}|x|$ implies $\rho_{*} \geq C^{-1} \sqrt{\frac{\gamma}{n}}$. This proves (vi).

Completion of proof of Lemma 1.1. We need to define $\alpha$ and $\beta$ and prove (ii)-(iv). We can take $\alpha=\frac{7}{2} \epsilon, \beta=C \sqrt{\gamma}+2 \sqrt{\delta}$ where $\mathrm{C}$ is the constant in Lemma 1.7. Then (ii) is proved as follows: $\operatorname{supp}\left(\triangle u_{n}\right) \subset B \cup B_{*}$ where $B=\{x:|r-1| \leq 3 \epsilon\} \cap\left\{x: \rho \leq 2 \sqrt{\frac{\delta}{n}}\right\}$ and $B_{*}$ is defined similarly replacing $r$ and $\rho$ by $r_{*}$ and $\rho_{*}$. If $\mathrm{n}$ is large, then (ii) follows using (i) and (iii) of Lemma 1.7 .

Next (i) and (ii) of Lemma 1.7 imply

$$
\begin{gathered}
\left|r^{n}-r_{*}^{n}\right| \lesssim \gamma r^{n} \\
\left|e^{i n \theta}-e^{i n \theta_{*}}\right| \lesssim \gamma
\end{gathered}
$$

on $A_{n}$, provided we have chosen $\delta<\gamma$.

We now prove (iii). Constants in this argument may depend on everything except $n$. We have

$$
\begin{aligned}
& \triangle\left(\chi_{n}(r, \rho) a_{n}(r, \rho) r^{n} \cos n \theta\right) \\
= & \triangle\left(\chi_{n}(r, \rho)\right) a_{n}(r, \rho) r^{n} \cos (n \theta)+2 \nabla\left(\chi_{n}(r, \rho)\right) \cdot \nabla\left(a_{n}(r, \rho) r^{n} \cos (n \theta)\right) \\
= & \triangle\left(\chi_{n}(r, \rho)\right) a_{n}(r, \rho) r^{n} \cos n \theta+2 \nabla\left(\chi_{n}(r, \rho)\right) \cdot \nabla\left(a_{n}(r, \rho)\right) r^{n} \cos n \theta \\
& +2 a_{n}(r, \rho) \frac{\partial \chi_{n}}{\partial r}(r, \rho) n r^{n-1} \cos n \theta .
\end{aligned}
$$

The various terms here may be bounded by $n r^{n}$ using the derivative bounds on $p$ and $q_{n}$ and the estimates in Lemma 1.2 (iii). Namely, $\left|\triangle \chi_{n}\right| \lesssim n$ and $\left|a_{n}\right| \lesssim 1$ on $R_{n}$ (note that $R_{n}$ contains the set where $\chi_{n} \neq 0$ ), so the first term is $\lesssim n r^{n}$. Likewise $\left|\nabla \chi_{n}\right| \lesssim n^{\frac{1}{2}}$, and $\left|\nabla a_{n}\right| \lesssim n^{\frac{1}{2}}$ on $R_{n}$, so the second term is $\lesssim n r^{n}$. Finally the last term is $\lesssim n r^{n}$ since $\left|\frac{\partial \chi_{n}}{\partial r}\right| \lesssim 1$ and $\left|a_{n}\right| \lesssim 1$ on $R_{n}$. 
Similar estimates can of course be made for the second term in (19), so we conclude that

$$
\left|\triangle u_{n}\right| \lesssim r^{n}+r_{*}^{n}
$$

But this implies (iii) in view of (22).

To prove (iv) we first isolate the terms in $\nabla u_{n}$ where the derivative falls on $r^{n} \cos n \theta$ or $r_{*}^{n} \sin n \theta_{*}$, i.e.

$$
\begin{aligned}
\nabla u_{n}=\chi_{n}( & r, \rho) a_{n}(r, \rho) r^{n-1}\left(\cos n \theta \frac{\partial}{\partial r}-\sin n \theta \frac{1}{r} \frac{\partial}{\partial \theta}\right) \\
& \quad+\chi_{n}\left(r_{*}, \rho_{*}\right) a_{n}\left(r_{*}, \rho_{*}\right) r_{*}^{n-1}\left(\sin n \theta_{*} \frac{\partial}{\partial r_{*}}+\cos n \theta_{*} \frac{1}{r_{*}} \frac{\partial}{\partial \theta_{*}}\right)+E_{1}
\end{aligned}
$$

where

$$
E_{1}=\frac{1}{n}\left(\nabla\left(\chi_{n}(r, \rho) a_{n}(r, \rho)\right) r^{n} \cos n \theta+\nabla\left(\chi_{n}\left(r_{*}, \rho_{*}\right) a_{n}\left(r_{*}, \rho_{*}\right)\right) r_{*}^{n} \sin n \theta_{*}\right) .
$$

It is easily seen (using the bounds from Lemma 1.1 and the derivative bounds for $\chi_{n}$, as in the proof of (iii)) that $\left|E_{1}\right| \leq C(\delta, \epsilon) n^{-\frac{1}{2}}\left(r^{n}+r_{*}^{n}\right)$, and therefore $\left|E_{1}\right| \leq C(\delta, \epsilon) n^{-\frac{1}{2}} r^{n}$ by (22). Next, (22), (23), and (iv), (v) of Lemma 1.7 imply that

$$
\left|r_{*}^{n-1}\left(\sin n \theta_{*} \frac{\partial}{\partial r_{*}}+\cos n \theta_{*} \frac{1}{r_{*}} \frac{\partial}{\partial \theta_{*}}\right)-r^{n-1}\left(\sin n \theta \frac{\partial}{\partial r}+\cos n \theta \frac{1}{r} \frac{\partial}{\partial \theta}\right)\right| \leq C \gamma r^{n}
$$

with $C$ an absolute constant. We therefore obtain

$$
\begin{aligned}
\nabla u_{n}= & \chi_{n}(r, \rho) a_{n}(r, \rho) r^{n-1}\left(\cos n \theta \frac{\partial}{\partial r}-\sin n \theta \frac{1}{r} \frac{\partial}{\partial \theta}\right) \\
& +\chi_{n}\left(r_{*}, \rho_{*}\right) a_{n}\left(r_{*}, \rho *\right) r^{n-1}\left(\sin n \theta \frac{\partial}{\partial r}+\cos n \theta \frac{1}{r} \frac{\partial}{\partial \theta}\right)+E_{1}+E_{2}
\end{aligned}
$$

with $\left|E_{1}\right| \leq C(\delta, \epsilon) n^{-\frac{1}{2}} r^{n}$ and $\left|E_{2}\right| \leq C \gamma r^{n}\left|\chi_{n}\left(r_{*}, \rho_{*}\right) a_{n}\left(r_{*}, \rho_{*}\right)\right|$. By the triangle inequality

$$
\left|\nabla u_{n}\right| \geq J-C(\delta, \epsilon) n^{-\frac{1}{2}} r^{n}-C \gamma r^{n}\left|\chi_{n}\left(r_{*}, \rho_{*}\right) a_{n}\left(r_{*}, \rho_{*}\right)\right|
$$

where $J$, the absolute value of the first two groups of terms on the right side of (24), may be calculated explicitly using that $\sin ^{2} n \theta+\cos ^{2} n \theta=1$. This gives

$$
\begin{aligned}
J & =\sqrt{2} r^{n-1} \sqrt{\left(\chi_{n}(r, \rho) a_{n}(r, \rho)\right)^{2}+\left(\chi_{n}\left(r_{*}, \rho_{*}\right) a_{n}\left(r_{*}, \rho_{*}\right)\right)^{2}} \\
& \geq\left(\left|\chi_{n}(r, \rho) a_{n}(r, \rho)\right|+\left|\chi_{n}\left(r_{*}, \rho_{*}\right) a_{n}\left(r_{*}, \rho_{*}\right)\right|\right) r^{n-1} .
\end{aligned}
$$


Therefore, using the bounds for $E_{1}$ and $E_{2}$ and that $r \leq 2$,

$$
\begin{aligned}
\left|\nabla u_{n}\right| \geq\left(\frac { 1 } { 4 } \left(\left|\chi_{n}(r, \rho) a_{n}(r, \rho)\right|\right.\right. & \left.+\left|\chi_{n}\left(r_{*}, \rho_{*}\right) a_{n}\left(r_{*}, \rho_{*}\right)\right|\right) \\
& \left.-C \gamma\left|\chi_{n}\left(r_{*}, \rho_{*}\right) a_{n}\left(r_{*}, \rho_{*}\right)\right|-C(\delta, \epsilon) n^{-\frac{1}{2}}\right) r^{n}
\end{aligned}
$$

The term involving $\gamma$ may be dropped if $\gamma$ has been chosen sufficiently small. Furthermore estimate (9) implies that $\left|\chi_{n}(r, \rho) a_{n}(r, \rho)\right|+\left|\chi_{n}\left(r_{*}, \rho_{*}\right) a_{n}\left(r_{*}, \rho_{*}\right)\right| \geq$ $\frac{1}{2}$ provided $\delta$ is small enough, since then either $\rho$ or $\rho_{*}$ will be $\geq 2\left(\frac{\delta}{n}\right)^{\frac{1}{2}}$ by Lemma 1.7(vi). Estimate (iv) now follows by taking $\mathrm{n}$ sufficiently large.

\section{Proof of Theorem 1, Part 2.}

We first prove a certain genericity statement. Denote

$\mathcal{H}_{k}=$ homogeneous harmonic polynomials of degree $\mathrm{k}$

$\mathcal{H}_{k}^{*}=\left\{Y \in \mathcal{H}_{k}: \nabla Y\right.$ vanishes only at 0$\}$

Let $\Gamma^{e}(x)=|x-e|^{2-d}$, let $e_{1}$ be the first standard basis vector and $Z_{k}$ (a normalization of the kth zonal harmonic) the degree $\mathrm{k}$ term in the Taylor expansion of $\Gamma^{e_{1}}$ at 0 . Let $\mathcal{O}(d)$ be the orthogonal group. If $\rho \in \mathcal{O}(d)$ maps $e$ to $e_{1}$ then $\Gamma^{e}=\Gamma^{e_{1}} \circ \rho$, so $Z_{k} \circ \rho$ is the degree $\mathrm{k}$ term in the expansion of $\Gamma^{e}$ at 0 .

Lemma 2.1. If $Y \in \mathcal{H}_{k}$ then the set $\left\{\left(\rho_{1}, \ldots, \rho_{d}, \alpha\right) \in \mathcal{O}(d) \times \ldots \times \mathcal{O}(d) \times \mathbb{R}^{d}\right.$ : $\left.Y+\sum_{j} \alpha_{j} Z_{k} \circ \rho_{j} \in \mathcal{H}_{k}^{*}\right\}$ has full measure in $\mathcal{O}(d) \times \ldots \times \mathcal{O}(d) \times \mathbb{R}^{d}$.

Proof. We first show that the set $E=\left\{\left(\rho_{1}, \ldots, \rho_{d}\right) \in \mathcal{O}(d) \times \ldots \times \mathcal{O}(d)\right.$ : $Z_{k} \circ \rho_{1}, \ldots, Z_{k} \circ \rho_{d}$ have no common zeroes except the origin $\}$ has full measure.

For this, consider the map

$$
\begin{gathered}
F: \mathcal{O}(d) \times \ldots \times \mathcal{O}(d) \times S^{d-1} \rightarrow \mathbb{R}^{d} \\
F\left(\rho_{1}, \ldots, \rho_{d}, x\right)=Z_{k}\left(\rho_{1} x\right), \ldots, Z_{k}\left(\rho_{d} x\right)
\end{gathered}
$$

For $i, j \in\{1, \ldots, d\}$ the $\rho_{i}$ - derivative of $Z_{k}\left(\rho_{j} x\right)$ clearly vanishes identically if $i \neq j$, and if $i=j$ vanishes only when $\rho_{j} x$ is a critical point of $\left.Z_{k}\right|_{S^{d-1}}$. We conclude: if $\left(\rho_{1}, \ldots, \rho_{d}, x\right)$ is such that $\rho_{j} x$ is a regular point of $Z_{k}$ for all $j \in\{1, \ldots, d\}$, then $\left(\rho_{1}, \ldots, \rho_{d}, x\right)$ is a regular point of $F$. 
On the other hand 0 is a regular value of $\left.Z_{k}\right|_{S^{d-1}}$ by uniqueness for ODE's since $\left.Z_{k}\right|_{S^{d-1}}$ is a solution of a second order ODE in the variable $\angle \cdot 0 e_{1}$. So if $F\left(\rho_{1}, \ldots, \rho_{d}, x\right)=0$ then $\left(\rho_{1}, \ldots, \rho_{d}, x\right)$ is a regular point of $F$, i.e. $F$ is transverse to zero. Define $F_{\rho_{1} \ldots \rho_{d}}: S^{d-1} \rightarrow \mathbb{R}^{d}$ by $F_{\rho_{1} \ldots \rho_{d}}(x)=F\left(\rho_{1}, \ldots, \rho_{d}, x\right)$. By the transversality theorem 0 is a regular value of $F_{\rho_{1} \ldots \rho_{d}}$ for a.e. $\left(\rho_{1}, \ldots, \rho_{d}\right)$. But by dimensional considerations 0 can only be a regular value of $F_{\rho_{1} \ldots \rho_{d}}$ if it is an omitted value. This proves the claim.

If $\left(\rho_{1}, \ldots, \rho_{d}\right) \in E$ then the function

$$
\begin{gathered}
G: \mathbb{R}^{d} \times S^{d-1} \rightarrow \mathbb{R}^{d}, \\
G(\alpha, x)=Y(x)+\sum_{j} \alpha_{j} Z_{k}\left(\rho_{j} x\right)
\end{gathered}
$$

is transverse to zero, since its $\alpha_{j}$ derivative is $Z_{k}\left(\rho_{j} x\right)$. So 0 is a regular value of the function $G_{\alpha}: S^{d-1} \rightarrow \mathbb{R}, G_{\alpha}(x)=Y(x)+\sum_{j} \alpha_{j} Z_{k}\left(\rho_{j} x\right)$, for a full measure set of $\alpha$. But if 0 is a regular value of $G_{\alpha}$ on $S^{d-1}$ then (by Euler's identity for homogeneous functions) $Y+\sum_{j} \alpha_{j} Z_{k} \circ \rho_{j}$ has no critical points in $\mathbb{R}^{d} \backslash 0$. This finishes the proof.

We will now make a certain technical modification in Lemma 1.1. Let $A_{n}$ be as there.

Lemma 2.2. Assume $d \geq 4$. Then there is $n_{0}<\infty$ and a sequence of smooth functions $u_{n}: \mathbb{R}^{d} \rightarrow \mathbb{R}\left(n_{0} \leq n<\infty\right)$ such that

(i) $u_{n}=\mathcal{O}\left(|x|^{n}\right)$ at $\infty, u_{n}=\mathcal{O}\left(|x|^{n+1}\right)$ at 0 . Furthermore let $p_{n}$ and $q_{n}$ be respectively the degree $n$ term in the expansion of $u_{n}$ at $\infty$ and the degree $n+1$ term in the Taylor expansion at 0 . Then $p_{n} \in \mathcal{H}_{n}^{*}$ and $q_{n}=p_{n+1}$.

(ii) $\operatorname{supp}\left(\triangle u_{n}\right) \subset A_{n}$.

(iii) $\left|\triangle u_{n}\right| \lesssim \eta_{n} r^{n}$ and $\left|\nabla u_{n}\right| \gtrsim \eta_{n} r^{n}$ on $A_{n}$, where the $\left\{\eta_{n}\right\}$ are constants.

Remark. Perhaps we should be more precise about the definition of the function $p_{n}$. If $\mathrm{u}$ is a harmonic function defined on the complement of a compact set in $\mathbb{R}^{d}$ with $d \geq 4$ and $u(x)=\mathcal{O}\left(|x|^{n}\right)$ at $\infty$, for some $n$ then it is not hard to show the 
Claim 2.3. There is a harmonic polynomial $p$ such that $u-p$ is $\mathcal{O}\left(|x|^{2-d}\right)$ at $\infty$.

Given the claim, we may let $p_{n}$ be the terms of degree $\mathrm{n}$ in the polynomial $p$. The claim is probably fairly well known but we will sketch the proof since we do not know a reference. We use induction on $n$, where $n$ is the smallest integer such that $u(x)=\mathcal{O}\left(|x|^{n}\right)$ at $\infty$. If $n \leq 0$ the statement is standard and follows from the removable singularities theorem for harmonic functions by using the Kelvin transform. Now suppose $n \geq 1$ and $u(x)=\mathcal{O}\left(|x|^{n}\right)$ at $\infty$. Then $\nabla u(x)=\mathcal{O}\left(|x|^{n-1}\right)$ so may be assumed to have the form of the claim. Now write $u(x)=\int_{x_{0}}^{x} \nabla u \cdot d x+u\left(x_{0}\right), x$ large, for a fixed $x_{0} \neq 0$. The assumption $d \geq 4$ implies that $|x|^{2-d}$ is integrable on rays not containing the origin. It follows that $u(x)$ has the form polynomial + bounded and then the claim follows from the $n=0$ case.

Proof of Lemma 2.2. We claim first that Lemma 1.1 is valid with the additional conclusion that the degree $n$ term in $u_{n}$ at $\infty$ and degree $n+2$ term at 0 belong to $\mathcal{H}_{n}^{*}$ and $\mathcal{H}_{n+2}^{*}$ respectively.

For this, let $u_{n}$ be the function from Lemma 1.1 and $p_{n}$ and $q_{n}$ its degree $\mathrm{n}$ term at $\infty$ and degree $n+2$ term at 0 . By Lemma 2.1 the set

$$
\begin{aligned}
& \left\{\left(\rho_{1}, \ldots, \rho_{d}, \alpha \in \mathcal{O}(d) \times \ldots \times \mathcal{O}(d) \times \mathbb{R}^{d}:\right.\right. \\
& \left.p_{n}-\sum_{j=1}^{d} \alpha_{j} Z_{n} \circ \rho_{j} \in \mathcal{H}_{n}^{*}, q_{n}+\sum_{j=1}^{d} \alpha_{j} Z_{n} \circ \rho_{j} \in \mathcal{H}_{n+2}^{*}\right\}
\end{aligned}
$$

has full measure. Hence, letting $Z_{k}^{e}$ be the degree $k$ term in $\Gamma^{e}$ at 0 , the set

$$
\begin{aligned}
& J=\left\{\left(x_{1}, \ldots, x_{d}, \alpha\right) \in S^{d-1} \times \ldots \times S^{d-1} \times \mathbb{R}^{d}:\right. \\
& \left.p_{n}-\sum_{j=1}^{d} \alpha_{j} Z_{n}^{x_{j}} \in \mathcal{H}_{n}^{*}, q_{n}+\sum_{j=1}^{d} \alpha_{j} Z_{n+2}^{x_{j}} \in \mathcal{H}_{n+2}^{*}\right\}
\end{aligned}
$$

has full measure.

If $\mathrm{n}$ is even, then we choose $\left\{x_{j}\right\}_{j=1}^{d} \subset A_{n}$ so that $\left(x_{1}, \ldots x_{d}, \alpha\right) \in J$ for a full measure set of $\alpha \in \mathbb{R}^{d}$. Let $\tilde{\Gamma}_{x_{j}}$ be a smooth function which agrees with $\Gamma_{x_{j}}$ outside $A_{n}$, let $T_{x_{j}}^{(n)}$ be the degree $\mathrm{n}$ Taylor polynomial of $\Gamma_{x_{j}}$ at 0 and 
consider the function

$$
\tilde{u_{n}}(x)=u_{n}(x)+\frac{1}{2} \sum_{j=1}^{d} \alpha_{j}\left[\tilde{\Gamma}_{x_{j}}(x)-T_{x_{j}}^{(n)}(x)+\tilde{\Gamma}_{x_{j}}(-x)-T_{x_{j}}^{(n)}(-x)\right]
$$

where the $\alpha_{j}$ are very small and $\left(x_{1}, \ldots, x_{d}, \alpha\right) \in J$. If the $\alpha_{j}$ are small enough then clearly (iii) and (iv) of Lemma 1.1 will still hold. Moreover $\tilde{u}_{n}$ is $\mathcal{O}\left(|x|^{n}\right)$ at $\infty, \mathcal{O}\left(|x|^{n+1}\right)$ at 0 , and being even, must in fact be $\mathcal{O}\left(|x|^{n+2}\right)$ at 0 . The degree $\mathrm{n}$ term at $\infty$ and degree $\mathrm{n}+2$ term at 0 are respectively $p_{n}-\sum_{j=1}^{d} \alpha_{j} Z_{n}^{x_{j}}$ and $q_{n}+\sum_{j=1}^{d} \alpha_{j} Z_{n+2}^{x_{j}}$ and therefore belong to $\mathcal{H}_{n}^{*}$ and $\mathcal{H}_{n+2}^{*}$ by construction. This proves the claim for $\mathrm{n}$ even. If $\mathrm{n}$ is odd we define instead

$$
\tilde{u}_{n}(x)=u_{n}(x)+\frac{1}{2} \sum_{j=1}^{d} \alpha_{j}\left[\tilde{\Gamma}_{x_{j}}(x)-T_{x_{j}}^{(n)}(x)-\left(\tilde{\Gamma}_{x_{j}}(-x)-T_{x_{j}}^{(n)}(-x)\right)\right]
$$

and proceed the same.

To finish the proof of the lemma let $u_{n}$ be as in the claim and denote its degree $n$ term at $\infty$ and degree $n+2$ term at 0 by $a_{n}$ and $b_{n}$ respectively. Let $v_{n}=u_{n}-a_{n}$ which is $\mathcal{O}\left(|x|^{n-2}\right)$ at $\infty, \mathcal{O}\left(|x|^{n}\right)$ at 0 , with the order $\mathrm{n}$ term at 0 being $-a_{n}$. Consider the functions $w_{n}=u_{n}-\epsilon_{n} v_{n+1}$ where $\epsilon_{n}$ is a small positive constant. $w_{n}$ is $\mathcal{O}\left(|x|^{n}\right)$ at $\infty, \mathcal{O}\left(|x|^{n+1}\right)$ at 0 , with the order $n$ term at $\infty$ being $a_{n}$ and the order $n+1$ term at 0 being $\epsilon_{n} a_{n+1}$. Also supp $\triangle w_{n} \subset A_{n} \cup A_{n+1}=A_{n}$, and if $\epsilon_{n}$ is small then clearly (iii) and (iv) of Lemma 1.1 will hold for $w_{n}$.

Now define the function $u_{n}$ of Lemma 2.2 by $u_{n}=\left(\prod_{j<n} \epsilon_{j}\right) \cdot w_{n}$. Since $u_{n}$ is a scalar multiple of $w_{n}$ it has properties (ii) and (iii) of Lemma 2.2 and is $\mathcal{O}\left(|x|^{n}\right)$ at $\infty, \mathcal{O}\left(|x|^{n+1}\right)$ at 0 . The order $n+1$ term in $u_{n}$ at 0 and order $n+1$ term in $u_{n+1}$ at $\infty$ are both equal to $\left(\prod_{j<n+1} \epsilon_{j}\right) \cdot a_{n+1}$ so the proof is complete.

We will now proceed more or less as in our earlier paper [7], although the details are simpler in the present context.

Lemma 2.4 (gluing lemma). Assume $d \geq 4$. Let $u_{n}$ be as in Lemma 2.2 and suppose $\epsilon>0$ is given. Then if $s>0$ is small enough and $r<\infty$ large enough there is a function $g_{n}: \mathbb{R}^{d} \rightarrow \mathbb{R}$ such that

(i) $g_{n}(x)=r^{n+1} u_{n}\left(r^{-1} x\right)$ if $|x| \geq 2$. 
(ii) $g_{n}(x)=s^{n+1} u_{n+1}\left(s^{-1} x\right)$ if $|x| \leq \frac{1}{2}$.

(iii) $\left|\triangle g_{n}\right| \leq \epsilon\left|\nabla g_{n}\right|$ if $\frac{1}{2} \leq|x| \leq 2$.

Furthermore $g_{n}$ satisfies bounds $\left|D^{\alpha} g_{n}\right| \leq C_{\alpha, n}|x|^{n+1-|\alpha|}$ independently of $r$ and $s$ provided $r$ and $s^{-1}$ are sufficiently large.

Proof. Simply choose a smooth function $\psi$ with $\psi=1$ when $|x| \leq \frac{1}{2}, \psi=0$ when $x \geq 2$ and define

$$
g_{n}(x)=\psi(x) s^{n} u_{n+1}\left(s^{-1} x\right)+(1-\psi(x)) r^{n} u_{n}\left(r^{-1} x\right) .
$$

We need only prove the estimates. However, in the region $\frac{1}{4} \leq|x| \leq 4$ we have

$$
\left|r^{n+1} u_{n}\left(r^{-1} x\right)-q_{n}(x)\right| \leq C_{n} r^{-1}
$$

since $u_{n}-q_{n}=\mathcal{O}\left(|x|^{n+2}\right)$ at 0 . By the derivative estimates for harmonic functions we also have

$$
\left|D^{\alpha}\left(r^{n+1} u_{n}\left(r^{-1} x\right)-q_{n}(x)\right)\right| \leq C_{\alpha, n} r^{-1}
$$

when $\frac{1}{2} \leq x \leq 2$, for any $\alpha$. Using that $u_{n+1}-p_{n+1}=\mathcal{O}\left(|x|^{n}\right)$ at $\infty$ and arguing in the same way we obtain a similar bound

$$
\left|D^{\alpha}\left(s^{n+1} u_{n+1}\left(s^{-1} x\right)-p_{n+1}(x)\right)\right| \leq C_{\alpha, n} s, \quad \frac{1}{2} \leq|x| \leq 2 .
$$

Since $p_{n+1}=q_{n}$ this may of course be written as

$$
\left|D^{\alpha}\left(s^{n+1} u_{n+1}\left(s^{-1} x\right)-q_{n}(x)\right)\right| \leq C_{\alpha, n} s .
$$

By (25) and (26)

$$
\begin{aligned}
& \left|\nabla g_{n}\right| \geq\left|\nabla q_{n}\right|-C_{\alpha, n}\left(r^{-1}+s\right) \\
& \left|\triangle g_{n}\right| \leq C_{\alpha, n}\left(r^{-1}+s\right)
\end{aligned}
$$

when $\frac{1}{2} \leq x \leq 2$. Since $q_{n} \in \mathcal{H}_{n+1}^{*}$ the bound (iii) now follows immediately. Next by (i) of Lemma 2.2 and derivative estimates for harmonic functions we have $\left|D^{\alpha} u_{n}(x)\right| \leq C_{\alpha, n}|x|^{n+1-|\alpha|}$ for small $|x|,\left|D^{\alpha} u_{n+1}(x)\right| \leq C_{\alpha, n}|x|^{n+1-|\alpha|}$ for large $|x|$. The last statement in the lemma follows by scaling arguments, which we omit. 
Proof of Theorem 1. Let $\left\{r_{n}\right\}_{n=n_{0}}^{\infty}$ be a sequence decreasing rapidly to zero. Define $g_{n}$ using Lemma 2.4 with $\epsilon=2^{-n}, r=\sqrt{\frac{r_{n}}{r_{n+1}}}, s=\sqrt{\frac{r_{n+1}}{r_{n}}}$ and let $h_{n}(x)=\left(\sqrt{r_{n} r_{n+1}}\right)^{n+1} g_{n}\left(\frac{x}{\sqrt{r_{n} r_{n+1}}}\right)$. This is possible provided $r_{n+1}$ is small enough compared with $r_{n}$. Then

$$
\begin{array}{rlrl}
h_{n}(x)=r_{n}^{n+1} u_{n}\left(r_{n}^{-1} x\right), & |x| \geq 2 \sqrt{r_{n} r_{n+1}} \\
h_{n}(x)=r_{n+1}^{n+1} u_{n+1}\left(r_{n+1}^{-1} x\right), & |x| \leq \frac{1}{2} \sqrt{r_{n} r_{n+1}} \\
\left|\triangle h_{n}\right| \leq 2^{-n}\left(r_{n} r_{n+1}\right)^{-\frac{1}{2}}\left|\nabla h_{n}\right|, \frac{1}{2} \sqrt{r_{n} r_{n+1}} \leq x \leq 2 \sqrt{r_{n} r_{n+1}} .
\end{array}
$$

The derivative bounds in Lemma 2.4 translate to bounds $\left|D^{\alpha} h_{n}\right| \leq C_{\alpha, n}|x|^{n+1-|\alpha|}$.

Define the function $u$ of Theorem 1 by

$$
\begin{aligned}
& u(x)=r_{n_{0}}^{-1} h_{n_{0}}(x) \quad \text { if }|x|>r_{n_{0}} \\
& u(x)=\left(\prod_{j \leq n} r_{j}\right)^{-1} \cdot h_{n}(x) \quad \text { if } n \geq n_{0}, r_{n+1}<|x| \leq r_{n} .
\end{aligned}
$$

Then $u$ is smooth on the boundaries $|x|=r_{n}$, since when $|x| \in\left(2 \sqrt{r_{n} r_{n+1}}, r_{n}\right)$ we have $u(x)=\left(\prod_{j \leq n} r_{j}\right)^{-1} \cdot r_{n}^{n+1} u_{n}\left(r_{n}^{-1} x\right)$ and when $|x| \in\left(r_{n}, 2 \sqrt{r_{n-1} r_{n}}\right)$ we have $u(x)=\left(\prod_{j \leq n-1} r_{j}\right)^{-1} \cdot r_{n}^{n} u_{n}\left(r_{n}^{-1} x\right)$ which is the same.

Furthermore all derivatives of $u$ go to zero as $|x| \rightarrow 0$ provided the $\left\{r_{j}\right\}$ decrease fast enough. Namely, the derivative bounds on the $h_{n}$ translate to bounds

$$
\left|D^{\alpha} u(x)\right| \leq C_{\alpha, n}\left(\prod_{j \leq n} r_{j}\right)^{-1} \cdot|x|^{n+1-|\alpha|}, \quad r_{n+1} \leq|x| \leq r_{n} .
$$

If $n \geq|\alpha|$ this is $\leq C_{\alpha, n}\left(\prod_{j \leq n} r_{j}\right)^{-1} r_{n}^{n+1-|\alpha|}=C_{\alpha, n}\left(\prod_{j<|\alpha|} r_{j}\right)^{-1} \prod_{|\alpha| \leq j \leq n} \frac{r_{n}}{r_{j}}$ which goes to zero as $n \rightarrow \infty$ for all $\alpha$ provided (say) $\frac{r_{n}}{r_{n-1}} \leq \min _{\alpha:|\alpha| \leq \frac{n}{2}} C_{\alpha, n}^{-1} \prod_{j \leq|\alpha|} r_{j}$.

Next we show that $|\triangle u| \leq C|x|^{-1}|\nabla u|$. Define $B_{n}=\left\{x: \frac{1}{2} \sqrt{r_{n} r_{n+1}}<\right.$ $\left.|x|<2 \sqrt{r_{n} r_{n+1}}\right\}$. Then $\triangle u$ vanishes except on the sets $r_{n} A_{n}$ and the sets $B_{n}$. If $x \in B_{n}$ then $|\triangle u| \leq 2^{-n}|x|^{-1}|\nabla u|$ by (27). On the other hand, if $x \in r_{n} A_{n}$ then, using scaling and (iii) of Lemma 2.2, we obtain $\frac{|\Delta u(x)|}{|\nabla u(x)|}=r_{n}^{-1} \frac{\left|\Delta u_{n}\left(r_{n}^{-1} x\right)\right|}{\left|\nabla u_{n}\left(r_{n}^{-1} x\right)\right|} \leq$ $C r_{n}^{-1}$, and therefore $|\Delta u(x)| \leq C|x|^{-1}|\nabla u(x)|$, as claimed. 
This completes the proof of part (a) of Theorem 1. Now we prove (b). Namely,

$$
\begin{aligned}
\left\|\frac{\Delta u}{|\nabla u|}\right\|_{d}^{d} & \lesssim \sum_{n=n_{0}}^{\infty} \int_{r_{n} A_{n}}|x|^{-d} d x+\sum_{n=n_{0}}^{\infty} \int_{B_{n}} 2^{-n d}|x|^{-d} d x \\
& \lesssim \sum_{n}\left|A_{n}\right|+\sum_{n} 2^{-n d} .
\end{aligned}
$$

The second sum is obviously finite, and $\left|A_{n}\right| \approx n^{-\left(\frac{d-2}{2}\right)}$ so the first sum is finite as well, provided $d \geq 5$.

We remark that in part (b) of the theorem, the $L^{d}$ norm of $V$ may be taken arbitrarily small: just start the construction at a high finite stage $n_{1}$ instead of at $n_{0}$.

\section{REFERENCES}

1. Barcelo B., Kenig, C., Ruiz, A., Sogge, C., Unique continuation properties for solutions of inequalities between the Laplacian and the gradient, Ill. J. Math. 32 (2) (1988), 230-245.

2. Jerison, D., Carleman inequalities for the Dirac and Laplace operators and unique continuation, Advan. Math. 62 (1986), 1118-134.

3. Jerison, D., Kenig, C., Unique continuation and absence of positive eigenvalues for Schrodinger operators, with an appendix by E. M. Stein, Ann. Math. 121 (1985), 463-494.

4. Pan, Y. F., Unique continuation for Schrodinger operators with singular potentials, Comm. PDE, to appear.

5. Plis, A., On nonuniqueness in Cauchy problems for an elliptic second order differential operator, Bull. Acad. Polon. Sci. 11 (1963), 95-100.

6. Wolff, T., Unique continuation for $|\triangle u| \leq V|\nabla u|$ and related problems, Revista Math. Iberoamericana 6:3-4(1990), 155-200.

7. Wolff, T., Note on counterexamples in strong unique continuation problems, Proc. Amer. Math. Soc. 114 (1992), 351-356.

8. Wolff, T., A property of measures in $\mathbb{R}^{n}$ and an application to unique continuation, Geometric and Functional Analysis 2 (1992), 225-284.

9. Wolff, T., Recent work on sharp estimates in second order elliptic unique continuation problems, Conference proceedings, Miraflores de la Sierra, 1992.

University of CaLifornia, Berkeley, U. S. A.

RECEIVED JUNE 22, 1993. 